\title{
Formation of Phosphorus-Nitrogen Bonds by Reduction of a Titanium Phosphine Complex Under Molecular Nitrogen
}

\author{
Lara Morello, Peihua Yu, Christopher D. Carmichael, Brian O. Patrick, and \\ Michael D. Fryzuk* \\ Department of Chemistry, The University of British Columbia, 2036 Main Mall, \\ Vancouver, BC, CANADA, V6T 1Z1
}

\section{Supporting Information}

\section{General Experimental Procedures}

All procedures and manipulations were performed under an atmosphere of dry, oxygen-free dinitrogen or argon by means of standard Schlenk or glovebox techniques (Vacuum Atmospheres HE-553-2 glovebox equipped with a MO-40-2H purification system and $\mathrm{a}-40^{\circ} \mathrm{C}$ freezer), unless otherwise specified. Argon and nitrogen were dried and deoxygenated by passing the gases through a column containing molecular sieves and MnO. Hexanes and toluene were purchased from Aldrich and dried by passage through a tower of alumina and degassed by passage through a tower of Q-5 catalyst under positive pressure of nitrogen. ${ }^{1}$ Anhydrous THF was pre-dried by refluxing over $\mathrm{CaH}_{2}$ and distilled from benzophenone ketyl ${ }^{2}$ under argon. Deuterated benzene was refluxed over sodium and potassium alloy under partial pressure, trap-to-trap distilled and freeze-pump-thaw degassed three times. ${ }^{1} \mathrm{H}$ and ${ }^{31} \mathrm{P}\left\{{ }^{1} \mathrm{H}\right\}$ NMR spectra were recorded on a Bruker AV-300 instrument operating at $300.1 \mathrm{MHz}$ for ${ }^{1} \mathrm{H}$ spectra. ${ }^{1} \mathrm{H}$ NMR spectra were referenced to residual protons in the deuterated solvent: $\mathrm{C}_{6} \mathrm{D}_{5} \mathrm{H}(7.15 \mathrm{ppm})$ and $\mathrm{C}_{7} \mathrm{D}_{7} \mathrm{H}(2.09 \mathrm{ppm}) .{ }^{31} \mathrm{P}\left\{{ }^{1} \mathrm{H}\right\} \mathrm{NMR}$ spectra were referenced to external $\mathrm{P}(\mathrm{OMe})_{3}\left(141.0 \mathrm{ppm}\right.$ with respect to $85 \% \mathrm{H}_{3} \mathrm{PO}_{4}$ at $\left.0.0 \mathrm{ppm}\right)$ or to a known impurity within the sample (e.g. 36.8 ppm singlet, $\left[\mathrm{P}_{2} \mathrm{~N}_{2}\right] \mathrm{H}_{2}$ ). All spectra were measured at room temperature.

Microanalyses $(\mathrm{C}, \mathrm{H}, \mathrm{N})$ were performed by Mr. P. Borda or Mr. M. Lakha and mass spectroscopy (low resolution EI) was performed by Mr. M. Lapawa; all of the University of British Columbia Department of Chemistry. 


\section{Materials}

Potassium graphite, $\mathrm{KC}_{8},{ }^{3,4} \quad \mathrm{ZrCl}_{4}(\mathrm{THF})_{2},{ }^{5} \quad \mathrm{TiCl}_{4}(\mathrm{THF})_{2},{ }^{5} \quad\left[\mathrm{P}_{2} \mathrm{~N}_{2}\right] \mathrm{Li}_{2}$ (dioxane), ${ }^{6}$ $[\mathrm{NPN}] \mathrm{Li}_{2}(\mathrm{THF})_{2},{ }^{7}$ were prepared according to reported literature methods. Neat $\mathrm{TiCl}_{4}$ was distilled and degassed by three freeze-pump-thaw cycles prior to use. A $1 \mathrm{~L}$ ampoule of 1 atm ${ }^{15} \mathrm{~N}_{2}$ was obtained from Cambridge Isotopes and fitted with a Kontes-valve adaptor.

\section{Synthesis and Characterization of $[\mathrm{NPN}] \mathrm{ZrCl}_{2}$ (THF)}

A sample of [NPN]Li ${ }_{2}(\mathrm{THF})_{2}(3.435 \mathrm{~g}, 5.795 \mathrm{mmol})$ was transferred to a thick-walled flask fitted with a Kontes valve. Toluene $(50 \mathrm{~mL})$ was added and the slurry was stirred until the white powder fully dissolved. A sample of $\mathrm{ZrCl}_{4}(\mathrm{THF})_{2}(2.290 \mathrm{~g}, 6.071 \mathrm{mmol})$ was added to the toluene solution. Alternatively, the two powders could be mixed together prior to adding toluene. The headspace was evacuated and the pale-yellow/orange slurry was heated to $\sim 60^{\circ} \mathrm{C}$ and stirred for a minimum of 24 hours. The flask was allowed to cool and the cream coloured slurry was filtered through a scintered-glass crucible containing Celite. The resulting clear, pale yellow solution was concentrated by removing much of the solvent in vacuo. With the addition of hexanes $(\sim 15 \mathrm{~mL})$, a white powder precipitated out of the solution. The creamy-yellow powder was collected immediately and dried on a scinteredglass crucible by vacuum filtration. The product was isolated without the need for further purification. Yield $2.6 \mathrm{~g}, 68 \%$. More product was recovered from the yellow filtrate by completely removing the solvent in vacuo and washing the residues repeatedly with hexanes.

${ }^{1} \mathbf{H}$ NMR $\left(\mathbf{C}_{6} \mathbf{D}_{6}, 300 \mathrm{MHz}\right): \delta-0.11\left(\mathrm{~s}, 6 \mathrm{H}, \mathrm{SiCH}_{3}\right), 0.25\left(\mathrm{~s}, 6 \mathrm{H}, \mathrm{SiCH}_{3}\right), 0.87(\mathrm{~m}, 4 \mathrm{H}, 3,4-$ H THF), 1.44 (m, 2H, $\left.\mathrm{CH}_{2}\right), 1.48$ (m, 2H, $\left.\mathrm{CH}_{2}\right), 3.35$ (m, 4H, 2,5-H THF), 6.84 (m, 2H, phenyl), 7.11 (m, 5H, phenyl), 7.22 (m, 2H, phenyl), 7.59 (m, 4H, phenyl), 8.28 (m, 2H, o-H P-phenyl).

${ }^{31} \mathbf{P}\left\{{ }^{1} \mathbf{H}\right\} \operatorname{NMR}\left(\mathbf{C}_{6} \mathbf{D}_{6}, 121 \mathrm{MHz}\right): \delta 1.67(\mathrm{~s})$.

\section{Synthesis and Characterization of $([\mathrm{NPN}] \mathrm{Zr}(\mathrm{THF}))_{2}\left(\mu-\eta^{2}: \eta^{2}-\mathrm{N}_{2}\right)$}

A thick-walled flask fitted with a Kontes valve was charged with [NPN] $\mathrm{ZrCl}_{2}(\mathrm{THF})$ (1.050 g, $1.570 \mathrm{mmol})$ and $\mathrm{KC}_{8}(0.437 \mathrm{~g}, 3.23 \mathrm{mmol})$ under an inert atmosphere. The 
reaction flask was then connected through a vacuum-transfer tube to another flask, also fitted with a Kontes valve, which contained degassed THF $(\sim 75 \mathrm{~mL})$. The contents of the reaction flask were placed under vacuum and stirred for 5-10 minutes. Degassed THF was added to the reaction flask by a trap-to-trap transfer under static vacuum at $-196^{\circ} \mathrm{C}$. After transferring all of the solvent, the liquid nitrogen bath was removed and the reaction flask was warmed to $-78^{\circ} \mathrm{C}$ (IPA/ $\mathrm{CO}_{2}$ bath) under a constant flow of $\mathrm{N}_{2}(1 \mathrm{~atm})$, while the contents were gently stirred. Once the solvent had completely melted, the flask was closed to $\mathrm{N}_{2}$ and the resulting brown slurry was stirred vigorously. The cold temperature was maintained for another 4-5 hours before the slurry was allowed to warm to R.T. and a colour change from brown to dark purple was observed. The dark purple slurry was stirred for another 24-48 hours. The reaction mixture was quickly filtered through Celite on a scintered-glass crucible and the solvent was removed in vacuo. A dark purple powder was collected from the residues. Yield $0.84 \mathrm{~g}, 87 \%$.

A mixture of products resulted from the reduction of $[\mathrm{NPN}] \mathrm{ZrCl}_{2}$ (THF) if the slurry was not stirred vigorously while warming to room temperature. A maroon coloured product and additional peaks in the ${ }^{31} \mathrm{P}\left\{{ }^{1} \mathrm{H}\right\}$ NMR spectrum indicates the presence of these impurities. The desired complex may be isolated, if necessary, by dissolving the crude solids in hexanes and allowing complex $[\mathrm{NPN}] \mathrm{ZrCl}_{2}$ (THF) to precipitate out of the slowly evaporating solution over several weeks.

Anal. Calcd. for $\mathrm{C}_{56} \mathrm{H}_{78} \mathrm{~N}_{6} \mathrm{O}_{2} \mathrm{P}_{2} \mathrm{Si}_{4} \mathrm{Zr}_{2}$ : C, 54.95; H, 6.42; N, 6.87. Found: C, 54.66; H, 6.47; $\mathrm{N}, 6.65$. EI-MS $m / z: 1080\left[\left([\mathrm{NPN}]_{2} \mathrm{Zr}_{2} \mathrm{~N}_{2}\right)^{+}\right]$

${ }^{1} \mathbf{H}$ NMR (C $\left.\mathbf{C}_{6} \mathbf{D}_{\mathbf{6}}, 300 \mathrm{MHz}\right): \delta 0.15$ (s, 12H, $\mathrm{SiCH}_{3}$ ), 0.55 (br.s, 20H, $\mathrm{SiCH}_{3}$ and 3,4-H THF), 1.44 (m, 4H, $\mathrm{CH}_{2}$ ), 1.66 (m, 4H, $\mathrm{CH}_{2}$ ), 3.21 (br.m, 8H, 2,5-H THF), 6.75 (m, 4H, m-H Pphenyl), 6.98 (m, 10H, phenyl), 7.13 (m, 12H, phenyl), 7.98 (m, 4H, o-H P-phenyl).

${ }^{31} \mathbf{P}\left\{{ }^{1} \mathbf{H}\right\}$ NMR $\left(\mathbf{C}_{6} \mathbf{D}_{6}, 121 \mathrm{MHz}\right): \delta-5.57$ (s).

\section{Synthesis and Characterization of $\left[\mathrm{P}_{2} \mathbf{N}_{2}\right] \mathrm{TiCl}_{2}$}

(i) Neat $\mathrm{TiCl}_{4}(1.5 \mathrm{~g}, 0.867 \mathrm{~mL}, 7.91 \mathrm{mmol})$ was added drop-wise to a clear colourless solution of $\left[\mathrm{P}_{2} \mathrm{~N}_{2}\right] \mathrm{Li}_{2}$ (dioxane) $(5.03 \mathrm{~g}, 7.92 \mathrm{mmol})$ in toluene $(40 \mathrm{~mL})$. The flask was sealed 
under an atmosphere of nitrogen and the resulting red opaque solution was stirred overnight. The red slurry was filtered through a sintered glass crucible containing Celite and the red filtrate was evaporated to dryness. A brick-red powder of $\left[\mathrm{P}_{2} \mathrm{~N}_{2}\right] \mathrm{TiCl}_{2}$ was isolated and washed with a toluene/hexanes solution to remove minor amounts of $\left[\mathrm{P}_{2} \mathrm{~N}_{2}\right] \mathrm{H}_{2}$. Crude yield $5.10 \mathrm{~g}, 99 \%$. Purified yield $3.94 \mathrm{~g}, 76 \%$.

(ii) A flask fitted with a Kontes valve was charged with $\left[\mathrm{P}_{2} \mathrm{~N}_{2}\right] \mathrm{Li}_{2}$ (dioxane) (1.37 g, 2.16 $\mathrm{mmol})$ and $\mathrm{TiCl}_{4}(\mathrm{THF})_{2}$ powder $(0.72 \mathrm{~g}, 2.16 \mathrm{mmol})$. Toluene $(50 \mathrm{~mL})$ was added to the yellow mixture. The headspace of the flask was evacuated and the dark red slurry was heated to reflux $\left(\sim 75^{\circ} \mathrm{C}\right)$ for 1.5 hours and stirred overnight at $50^{\circ} \mathrm{C}$. Upon cooling, the slurry was filtered through Celite, toluene was removed in vacuo, and a brick-red residue of $\mathbf{1 6}$ was isolated. Yield $0.98 \mathrm{~g}, 70 \%$.

Anal. Calcd. for $\mathrm{C}_{24} \mathrm{H}_{42} \mathrm{Cl}_{2} \mathrm{~N}_{2} \mathrm{P}_{2} \mathrm{Si}_{4} \mathrm{Ti}$ : C, 44.23; H, 6.50; N, 4.30. Found: C, 44.27; H, 6.56; $\mathrm{N}, 4.21$

${ }^{1} \mathbf{H}$ NMR $\left(\mathbf{C}_{6} \mathbf{D}_{6}, 300 \mathrm{MHz}\right): \delta 0.36$ (s, 12H, $\left.\mathrm{SiCH}_{3}\right), 0.40$ (s, 12H, $\mathrm{SiCH}_{3}$ ), 1.34 (br.m, 4H, $\mathrm{CH}_{2}$ ), 1.55 (br.m, 4H, $\mathrm{CH}_{2}$ ), 6.94 to 7.15 (overlapping m, 6H, phenyl), 7.88 (m, 4H, o- $\mathrm{H}$ phenyl).

${ }^{31} \mathbf{P}\left\{{ }^{1} \mathbf{H}\right\}$ NMR $\left(\mathbf{C}_{6} \mathbf{D}_{6}, 121 \mathrm{MHz}\right): \delta 0.8(\mathrm{~s})$.

\section{Synthesis and Characterization of $\left(\left[\mathbf{P}_{2} \mathbf{N}_{2}\right] \mathbf{T i}\right)_{2}\left(\mu-\eta^{1}: \eta^{1}-\mathbf{N}_{2}\right)$}

A thick-walled glass flask fitted with a Kontes valve was charged with a mixture of $\left[\mathrm{P}_{2} \mathrm{~N}_{2}\right] \mathrm{TiCl}_{2}(1.02 \mathrm{~g}, 1.57 \mathrm{mmol})$ and $\mathrm{KC}_{8}(0.45 \mathrm{~g}, 3.33 \mathrm{mmol})$. The mixture was stirred, cooled to $-78^{\circ} \mathrm{C}$ and exposed to a constant flow of $\mathrm{N}_{2}$ gas $(1 \mathrm{~atm})$. The mixture was stirred vigorously as THF $(40 \mathrm{~mL})$ was added via cannula. After stirring for 10-15 minutes the flask was sealed and the resulting red-brown slurry was kept cold for an additional 1-2 hours. The slurry slowly changed to yellow-brown as the reaction mixture warmed to room temperature and was stirred over night. The mixture was filtered through Celite to remove graphite. Toluene was removed in vacuo and a brown-green residue was isolated. Slow evaporation of a concentrated brown toluene solution of $\left\{\left[\mathrm{P}_{2} \mathrm{~N}_{2}\right] \mathrm{Ti}\right\}_{2}\left(\mu-\eta^{1}: \eta^{1}-\mathrm{N}_{2}\right)$ produced crystals suitable for single crystal X-ray diffraction. Yield 0.64g, 69\%. 
Anal. Calcd. for $\mathrm{C}_{48} \mathrm{H}_{84} \mathrm{~N}_{5} \mathrm{P}_{4} \mathrm{Si}_{8} \mathrm{Ti}_{2}$ : C, 48.04; H, 7.20; N, 5.96. Found: C, 49.04; H, 7.37; N, 5.88. EI-MS $m / z: 1188\left[\left(\left[\mathrm{P}_{2} \mathrm{~N}_{2}\right]_{2} \mathrm{Ti}_{2} \mathrm{~N}_{2}\right)^{+}\right]$

${ }^{1} \mathbf{H}$ NMR $\left(\mathbf{C}_{6} \mathbf{D}_{6}, 300 \mathrm{MHz}\right): \delta 0.14\left(\mathrm{~s}, 24 \mathrm{H}, \mathrm{SiCH}_{3}\right), 0.23\left(\mathrm{~s}, 24 \mathrm{H}, \mathrm{SiCH}_{3}\right), 1.23$ (broad overlapping $\mathrm{m}, 16 \mathrm{H}, \mathrm{CH}_{2}$ ), 6.89 to 7.27 (overlapping $\mathrm{m}, 12 \mathrm{H}$, phenyl), 8.16 (br.m, $8 \mathrm{H}, o-\mathrm{H}$ phenyl).

${ }^{31} \mathbf{P}\left\{{ }^{1} \mathrm{H}\right\}$ NMR $\left(\mathbf{C}_{6} \mathbf{D}_{6}, 121 \mathrm{MHz}\right): \delta-4.8(\mathrm{~s})$.

\section{Synthesis and Characterization of $[\mathrm{NPN}] \mathrm{TiCl}_{2}$}

Toluene $(30 \mathrm{~mL})$ was added to a flask containing a mixture of $\mathrm{TiCl}_{4}(\mathrm{THF})(1.750 \mathrm{~g}$, $5.24 \mathrm{mmol})$ and $[\mathrm{NPN}] \mathrm{Li}_{2}(\mathrm{THF})_{2}(3.103 \mathrm{~g}, 5.23 \mathrm{mmol})$. The dark red-orange slurry was stirred for at least one hour (or for up to one day) and then filtered through Celite to remove LiCl. The solvent was removed in vacuo, leaving a dark orange residue. Hexanes $(5 \mathrm{~mL})$ were added to partially solubilize the residues and then removed in vacuo until the product took on the appearance of orange powder. (Yield $1.74 \mathrm{~g}, 60 \%$ ). To further purify the sample the solids were scraped into a sintered glass funnel and washed with a minimal amount of toluene, followed by hexanes. The resulting orange solid was slightly paler than the original residues (Yield $1.50 \mathrm{~g}, 52 \%$ ). Red platelet crystals suitable for X-ray diffraction were grown by vapor diffusion of hexanes into a THF solution of $[\mathrm{NPN}] \mathrm{TiCl}_{2}$.

Anal. Calcd. for $\mathrm{C}_{24} \mathrm{H}_{31} \mathrm{Cl}_{2} \mathrm{~N}_{2} \mathrm{PSi}_{2}$ Ti: C, 52.09; H, 5.65; N, 5.06. Found: C, 52.46; H, 5.72; $\mathrm{N}$, 4.73. EI-MS $m / z: 552\left[\left([\mathrm{NPN}] \mathrm{TiCl}_{2}\right)^{+}\right]$

${ }^{1} \mathbf{H}$ NMR (C $\left.\mathbf{C}_{6} \mathbf{D}_{6}, 300 \mathrm{MHz}\right): \delta-0.13\left(\mathrm{~s}, 6 \mathrm{H}, \mathrm{SiCH}_{3}\right), 0.14\left(\mathrm{~s}, 6 \mathrm{H}, \mathrm{SiCH}_{3}\right), 1.21\left(\mathrm{~m}, 2 \mathrm{H}, \mathrm{CH}_{2}\right)$, 1.43 (m, 2H, $\mathrm{CH}_{2}$ ), 6.88 to 7.13 (overlapping m, 13H, N-phenyl and P-phenyl), 7.84 (m, 2H, $o$-H P-phenyl).

${ }^{31} \mathbf{P}\left\{{ }^{1} \mathrm{H}\right\}$ NMR $\left(\mathbf{C}_{6} \mathbf{D}_{6}, 121 \mathrm{MHz}\right): \delta 3.7(\mathrm{~s})$.

\section{Synthesis and Characterization of ([N(PN)N]Ti) $)_{2}$; Reduction of $[\mathrm{NPN}] \mathrm{TiCl}_{2}$ under ${ }^{14} \mathrm{~N}_{2}$}

A thick-walled glass flask fitted with a Kontes valve was charged with a mixture of $[\mathrm{NPN}] \mathrm{TiCl}_{2}(0.147 \mathrm{~g}, 0.266 \mathrm{mmol})$ and $\mathrm{KC}_{8}(0.071 \mathrm{~g}, 0.526 \mathrm{mmol})$. The solids were stirred 
while under vacuum. Degassed THF $(40 \mathrm{~mL})$ was added in vacuo by a trap-to-trap transfer at $-196^{\circ} \mathrm{C}$. The frozen reaction mixture was flushed with $\mathrm{N}_{2}(1 \mathrm{~atm})$ while warmed to $-78^{\circ} \mathrm{C}$. The flask was sealed once the solvent was completely dissolved and the slurry was stirred at $-78^{\circ} \mathrm{C}$ for an additional 2-3 hours. The resulting dark brown/olive green slurry was stirred overnight while gradually warmed to room temperature. The mixture was filtered through Celite to remove graphite and solvent was removed in vacuo. The dark green residues (crude yield $0.107 \mathrm{~g}, 82 \%$ ) were dissolved in a minimal amount of toluene and set aside for slow evaporation. The olive green solution changed to emerald green over a period of weeks. Prismatic forest green crystals of $([\mathrm{N}(\mathrm{PN}) \mathrm{N}] \mathrm{Ti})_{2}$ were isolated and suitable for single crystal X-ray diffraction. Crystal yield $0.050 \mathrm{~g}, 38 \%$.

Anal. Calcd. for $\mathrm{C}_{48} \mathrm{H}_{62} \mathrm{~N}_{6} \mathrm{P}_{2} \mathrm{Si}_{4} \mathrm{Ti}_{2}$ : C, 58.05; H, 6.29; N, 8.46. Found: C, 57.72; H, 5.89; N, 8.86. EI-MS $m / z: 992\left[\left(\left[\mathrm{NN}^{\prime} \mathrm{N}_{2} \mathrm{Ti}_{2}\right)^{+}\right]\right.$

${ }^{1} \mathbf{H}$ NMR $\left(\mathbf{C}_{6} \mathbf{D}_{6}, 300 \mathrm{MHz}\right): \delta 0.13\left(\mathrm{~s}, 12 \mathrm{H}, \mathrm{SiCH}_{3}\right), 0.44\left(\mathrm{~s}, 12 \mathrm{H}, \mathrm{SiCH}_{3}\right), 0.89$ (m, 4H, $\mathrm{CH}_{2}$ ), 1.04 (m, 4H, $\left.\mathrm{CH}_{2}\right), 6.80$ (m, 1H, phenyl), 6.83 (br. m, 2H, phenyl), 6.85 (m, 1H, phenyl), 6.94 to 7.15 (overlapping $\mathrm{m}, 26 \mathrm{H}$, phenyl).

${ }^{31} \mathbf{P}\left\{{ }^{1} \mathbf{H}\right\}$ NMR (C $\left.\mathbf{D D}_{\mathbf{6}}, 121 \mathrm{MHz}\right): \delta 39.9$ (s).

\section{Reduction of $[\mathrm{NPN}] \mathrm{TiCl}_{2}$ under ${ }^{15} \mathrm{~N}_{2}$; Preparation of $\left(\left[\mathrm{N}\left(\mathrm{P}^{15} \mathrm{~N}\right) \mathrm{N}\right] \mathrm{Ti}\right)_{2}$}

Reduction of [NPN] TiCl $2(0.1334 \mathrm{~g}, 0.2410 \mathrm{mmol})$ with $\mathrm{KC}_{8}(0.0685 \mathrm{~g}, 0.507 \mathrm{mmol})$ under ${ }^{15} \mathrm{~N}_{2}$ was carried out in the same manner as the reduction under one atmosphere of ${ }^{14} \mathrm{~N}_{2}$ (i.e., vacuum transfer of THF, introduction of $\mathrm{N}_{2}$ at $-78^{\circ} \mathrm{C}$ ). The only exception being that the supply of ${ }^{15} \mathrm{~N}_{2}$ was limited; a 1L ampoule of ${ }^{15} \mathrm{~N}_{2}$ gas (1 atm) was used. The final pressure of ${ }^{15} \mathrm{~N}_{2}$ within the reaction vessel once warmed to room temperature was unknown. The resulting olive green slurry was stirred overnight at room temperature. The mixture was filtered through Celite and brown-green residues resulted from the removal of THF in vacuo. The ${ }^{1} \mathrm{H}$ NMR spectrum is complicated by many overlapping resonances. Attempts to obtain an ${ }^{15} \mathrm{~N}$ NMR spectrum were unsuccessful; a signal could not be detected, which is likely due to the small content of product in the sample.

EI-MS $m / z: 994\left[\left(\left[\mathrm{~N}\left(\mathrm{P}^{15} \mathrm{~N}\right) \mathrm{N}\right]_{2} \mathrm{Ti}_{2}\right)^{+}\right]$ 
${ }^{31} \mathbf{P}\left\{{ }^{1} \mathbf{H}\right\}$ NMR $\left(\mathbf{C}_{6} \mathbf{D}_{6}, 121 \mathrm{MHz}\right): \delta-41.6$ (s, relative integration area $\left.=23\right),-36.8(\mathrm{~s}$, relative integration area $=8,\left[\mathrm{NPN}_{3} \mathrm{H}_{2}\right),-34.1(\mathrm{~s}$, relative integration area $=3), 39.9\left(\mathrm{~d},{ }^{1} \mathrm{~J}_{\mathrm{PN}}\right.$ $22 \mathrm{~Hz}$, relative integration area $=10)$.

\section{X-Ray Crystallography}

A summary of all crystal data collection and refinement for compounds $\mathbf{A}, \mathbf{B}, \mathbf{C}$ and D is given in Table S1. Crystal structure data CIF files are available. Tables S2, S3, S4 and S5 contain selected bond lengths and angles for compounds $\mathbf{A}, \mathbf{B}, \mathbf{C}$ and $\mathbf{D}$, respectively.

All measurements were made on an ADSC CCD area detector coupled with a Rigaku AFC7 diffractometer with graphite monochromated Mo-K $\alpha$ radiation. Data were collected and processed using the $\mathrm{d}^{*}$ TREK program. ${ }^{8}$ The structures were solved by direct methods 9,10 and expanded using Fourier techniques. ${ }^{11}$ Hydrogen atoms were included but not refined. Non-hydrogen atoms were refined anisotropically.

No solvent molecules crystallize in the asymmetric unit with compound $\mathbf{A}$. Compound B crystallizes as two crystallographically independent half-molecules, each forming a $\mathrm{N}_{2}$-linked dimer via an inversion center. Compound $\mathbf{B}$ was found to crystallize with two molecules of toluene in the asymmetric unit. Compound $\mathbf{C}$ crystallizes with one uncoordinated molecule of THF in the asymmetric unit. Compound $\mathbf{D}$ crystallizes with two molecules of toluene in the asymmetric unit. 
Table S1. Summary of Crystallographic Data Collection and Refinement for $([\mathrm{NPN}] \mathrm{ZrTHF})_{2}\left(\mu-\eta^{2}: \eta^{2}-\mathrm{N}_{2}\right) \quad \mathbf{A}, \quad\left(\left[\mathrm{P}_{2} \mathrm{~N}_{2}\right] \mathrm{Ti}\right)_{2}\left(\mu-\eta^{1}: \eta^{1}-\mathrm{N}_{2}\right) \quad \mathbf{B}, \quad[\mathrm{NPN}] \mathrm{TiCl}_{2} \quad \mathbf{C}$, and $([\mathrm{N}(\mathrm{PN}) \mathrm{N}] \mathrm{Ti})_{2} \mathbf{D}$

\begin{tabular}{|c|c|c|c|c|}
\hline & $\mathbf{A}$ & B & C & $\mathbf{D}$ \\
\hline $\begin{array}{l}\text { Empirical } \\
\text { Formula }\end{array}$ & $\mathrm{C}_{56} \mathrm{H}_{78} \mathrm{~N}_{6} \mathrm{O}_{2} \mathrm{P}_{2} \mathrm{Si}_{4} \mathrm{Zr}_{2}$ & $\mathrm{C}_{62} \mathrm{H}_{100} \mathrm{~N}_{6} \mathrm{P}_{4} \mathrm{Si}_{8} \mathrm{Ti}_{2}$ & $\mathrm{C}_{28} \mathrm{H}_{39} \mathrm{~N}_{2} \mathrm{OPSi}_{2} \mathrm{Cl}_{2} \mathrm{Ti}$ & $\mathrm{C}_{62} \mathrm{H}_{78} \mathrm{~N}_{6} \mathrm{P}_{2} \mathrm{Si}_{4} \mathrm{Ti}_{2}$ \\
\hline FW & 1224.00 & 1373.88 & 625.56 & 1177.40 \\
\hline Colour, Habit & red, chip & brown, needle & red, platelet & green, block \\
\hline Crystal size, $\mathrm{mm}$ & $0.40 \times 0.40 \times 0.20$ & $0.40 \times 0.15 \times 0.10$ & $0.35 \times 0.15 \times 0.03$ & $0.35 \times 0.35 \times 0.2$ \\
\hline Crystal system & monoclinic & monoclinic & monoclinic & monoclinic \\
\hline Space group & $\mathrm{C} 2 / \mathrm{c}(\# 15)$ & C2/c (\#15) & $\mathrm{P} 2_{1}(\# 4)$ & $\mathrm{P} 2_{1} / \mathrm{n}$ \\
\hline $\mathrm{a}, \AA$ & $25.214(1)$ & $25.1300(9)$ & $8.6354(7)$ & $15.7640(9)$ \\
\hline $\mathrm{b}, \AA$ & $11.4673(4)$ & $26.7178(10)$ & $20.977(16)$ & $17.9296(10)$ \\
\hline c, $\AA$ & $21.2735(9)$ & $27.4226(10)$ & $8.8355(7)$ & $22.5620(14)$ \\
\hline$\alpha, \operatorname{deg}$ & 90.00 & 90.00 & 90.00 & 90.00 \\
\hline$\beta, \operatorname{deg}$ & $94.362(3)$ & $114.884(2)$ & $92.563(4)$ & $96.006(2)$ \\
\hline$\gamma, \operatorname{deg}$ & 90.00 & 90.00 & 90.00 & 90.00 \\
\hline $\mathrm{V}, \AA^{3}$ & $6133.1(4)$ & $16702.7(11)$ & $1598.9(2)$ & $6342.0(6)$ \\
\hline Z & 4 & 8 & 2 & 4 \\
\hline $\mathrm{T},{ }^{\circ} \mathrm{C}$ & $-100 \pm 1$ & $-100 \pm 1$ & $-100 \pm 1$ & $-100 \pm 1$ \\
\hline$\rho_{\text {calc }}, \mathrm{g} / \mathrm{cm}^{3}$ & 1.325 & 1.166 & 1.299 & 1.233 \\
\hline $\mathrm{F}_{000}$ & 2552.00 & 6240.00 & 656.00 & 2488 \\
\hline$\mu(\mathrm{MoK} \alpha), \mathrm{cm}^{-1}$ & 5.13 & 4.22 & 5.83 & 4.20 \\
\hline correction factors & $0.7611-1.0000$ & $0.6670-1.0000$ & $0.7207-1.0000$ & $0.8908-1.0000$ \\
\hline $2 \theta_{\max }, \operatorname{deg}$ & 60.1 & 53.1 & 55.7 & 55.8 \\
\hline total no. of reflns & 26425 & 65279 & 14981 & 55657 \\
\hline $\begin{array}{l}\text { no. of unique } \\
\text { reflns }\end{array}$ & 7492 & 16401 & 6616 & 14131 \\
\hline $\mathrm{R}_{\text {int }}$ & 0.047 & 0.083 & 0.056 & 0.0500 \\
\hline $\begin{array}{l}\text { no. observations } \\
(\mathrm{I}>\mathrm{n \sigma}(\mathrm{I}))\end{array}$ & $5333, \mathrm{n}=3$ & $9853, \mathrm{n}=2$ & $5130, \mathrm{n}=2$ & $11653, \mathrm{n}=2$ \\
\hline no. of variables & 343 & 708 & 343 & 671 \\
\hline $\mathrm{R} 1\left(\mathrm{~F}^{2}, \mathrm{I}>2 \sigma(\mathrm{I})\right)$ & 0.054 & 0.062 & 0.066 & 0.0595 \\
\hline $\begin{array}{l}\text { wR2 }\left(F^{2} \text {, all }\right. \\
\text { data) }\end{array}$ & 0.085 & 0.198 & 0.118 & 0.1442 \\
\hline Gof & 1.06 & 1.01 & 0.96 & 1.056 \\
\hline
\end{tabular}

Rigaku/ADSC CCD diffractometer

$\mathrm{R} 1=\Sigma|| \mathrm{F}_{\mathrm{o}}|-| \mathrm{F}_{\mathrm{c}}|| /\left|\mathrm{F}_{\mathrm{o}}\right| ; \mathrm{wR} 2=\left(\Sigma \mathrm{w}\left(\left|\mathrm{F}_{\mathrm{o}}{ }^{2}\right|-\left|\mathrm{F}_{\mathrm{c}}{ }^{2}\right|\right)^{2} / \Sigma \mathrm{w}\left|\mathrm{F}_{\mathrm{o}}{ }^{2}\right|^{2}\right)^{1 / 2}$ 


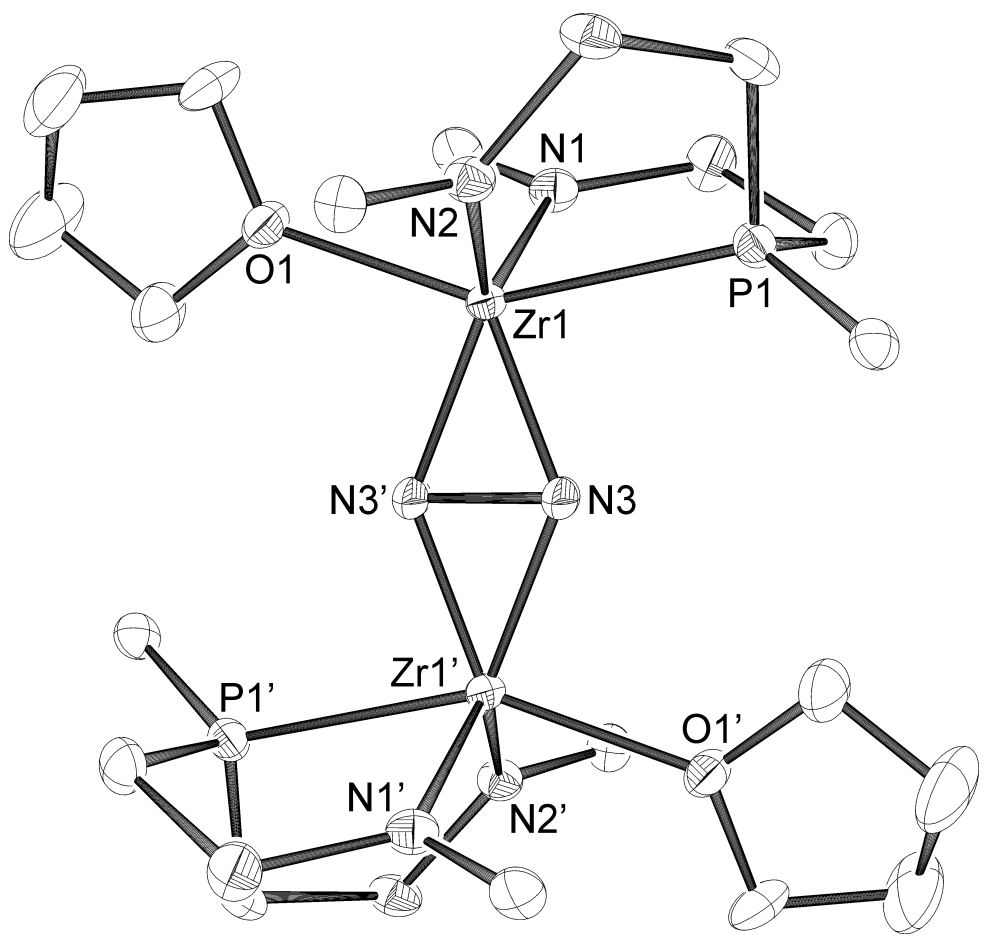

Figure S1. ORTEP representation (ellipsoid probability 50\%) of the molecular structure of $([\mathrm{NPN}] \mathrm{Zr}(\mathrm{THF}))_{2}\left(\mu-\eta^{2}: \eta^{2}-\mathrm{N}_{2}\right)$ as determined by X-ray diffraction. Hydrogen atoms and silylmethyl groups are omitted for clarity and only ipso carbons of phenyl rings are shown.

Table S2. Selected Bond Lengths and Angles in ([NPN]Zr(THF) $)_{2}\left(\mu-\eta^{2}: \eta^{2}-\mathrm{N}_{2}\right)$

\begin{tabular}{lll||lll}
\hline Atom & Atom & Distance $(\AA)$ & Atom & Atom & Distance $(\AA)$ \\
\hline $\mathrm{N}(3)$ & $\mathrm{N}(3)$ & $1.503(3)$ & $\operatorname{Zr}(1)$ & $\mathrm{N}(2)$ & $2.228(2)$ \\
$\mathrm{Zr}(1)$ & $\mathrm{N}(3)$ & $2.026(2)$ & $\operatorname{Zr}(1)$ & $\mathrm{O}(1)$ & $2.305(1)$ \\
$\mathrm{Zr}(1)$ & $\mathrm{N}(3)$ & $2.069(2)$ & $\operatorname{Zr}(1)$ & $\mathrm{P}(1)$ & $2.6685(5)$ \\
$\mathrm{Zr}(1)$ & $\mathrm{N}(1)$ & $2.175(2)$ & & & \\
\hline
\end{tabular}

\begin{tabular}{llll||llll}
\hline Atom & Atom & Atom & Angle $\left(^{\circ}\right)$ & Atom & Atom & Atom & Angle $\left(^{\circ}\right)$ \\
\hline $\mathrm{P}(1)$ & $\operatorname{Zr}(1)$ & $\mathrm{O}(1)$ & $149.24(4)$ & $\mathrm{N}(1)$ & $\operatorname{Zr}(1)$ & $\mathrm{N}(2)$ & $116.84(6)$ \\
$\mathrm{P}(1)$ & $\mathrm{Zr}(1)$ & $\mathrm{N}(2)$ & $77.15(5)$ & $\mathrm{N}(1)$ & $\operatorname{Zr}(1)$ & $\mathrm{N}(3)$ & $114.07(6)$ \\
$\mathrm{P}(1)$ & $\mathrm{Zr}(1)$ & $\mathrm{N}(1)$ & $76.62(5)$ & $\mathrm{N}(2)$ & $\operatorname{Zr}(1)$ & $\mathrm{N}(3)$ & $116.77(6)$ \\
$\mathrm{P}(1)$ & $\mathrm{Zr}(1)$ & $\mathrm{N}(3)$ & $80.73(5)$ & $\mathrm{O}(1)$ & $\operatorname{Zr}(1)$ & $\mathrm{N}(1)$ & $89.88(6)$ \\
$\mathrm{O}(1)$ & $\mathrm{Zr}(1)$ & $\mathrm{N}(2)$ & $84.90(5)$ & $\mathrm{O}(1)$ & $\operatorname{Zr}(1)$ & $\mathrm{N}(3)$ & $87.08(5)$ \\
\hline
\end{tabular}




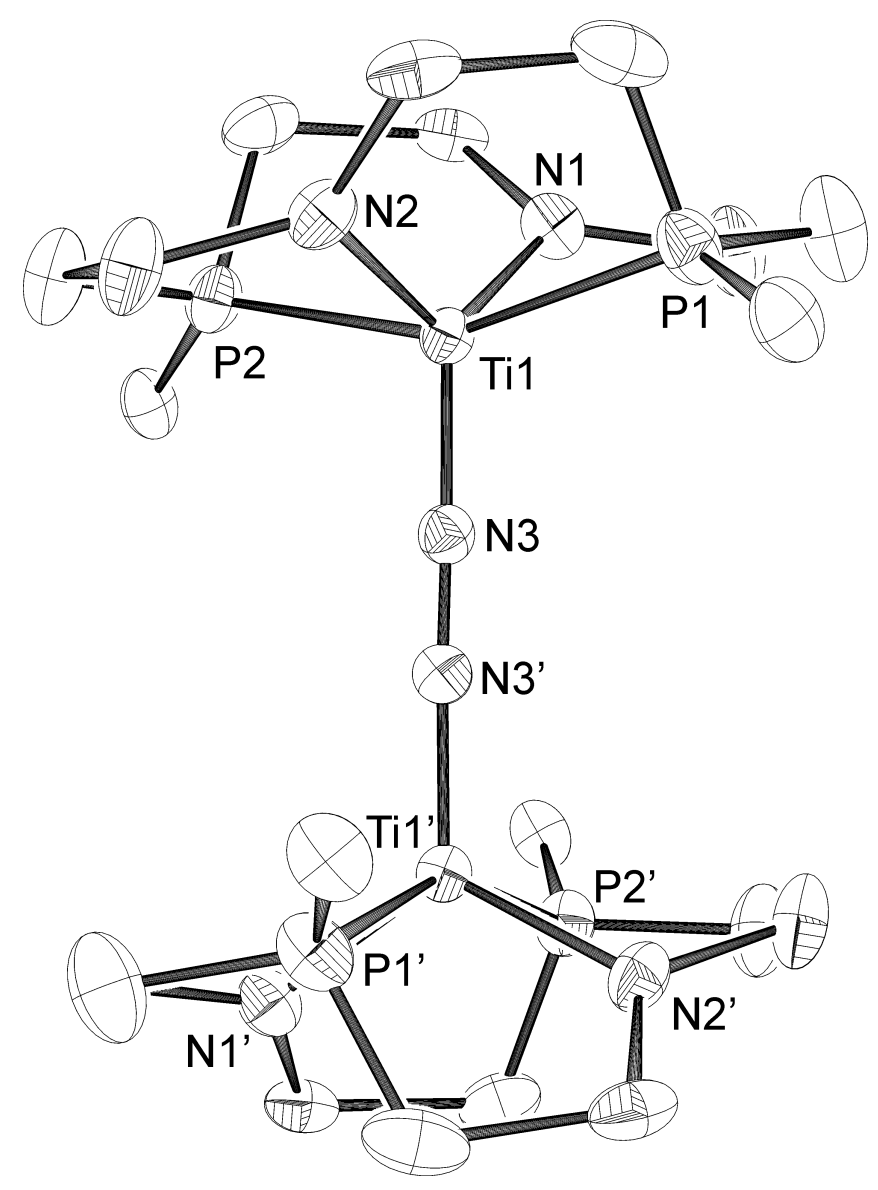

Figure S2. ORTEP representation (ellipsoid probability 50\%) of the molecular structure of $\left(\left[\mathrm{P}_{2} \mathrm{~N}_{2}\right] \mathrm{Ti}\right)_{2}\left(\mu-\eta^{1}: \eta^{1}-\mathrm{N}_{2}\right)$ as determined by X-ray diffraction. Hydrogen atoms and silylmethyl groups are omitted for clarity and only ipso carbons of phenyl rings are shown.

Table S3. Selected Bond Lengths and Angles in $\left(\left[\mathrm{P}_{2} \mathrm{~N}_{2}\right] \mathrm{Ti}\right)_{2}\left(\mu-\eta^{1}: \eta^{1}-\mathrm{N}_{2}\right)$

\begin{tabular}{lll||lll}
\hline Atom & Atom & Distance $(\AA)$ & Atom & Atom & Distance $(\AA)$ \\
\hline $\mathrm{N}(3)$ & $\mathrm{N}(3)$ & $1.255(7)$ & $\operatorname{Ti}(1)$ & $\mathrm{N}(3)$ & $1.783(4)$ \\
$\operatorname{Ti}(1)$ & $\mathrm{P}(1)$ & $2.5669(13)$ & $\operatorname{Ti}(1)$ & $\mathrm{N}(2)$ & $2.049(4)$ \\
$\operatorname{Ti}(1)$ & $\mathrm{P}(2)$ & $2.5552(13)$ & $\mathrm{Ti}(1)$ & $\mathrm{N}(1)$ & $2.076(4)$ \\
\hline
\end{tabular}

\begin{tabular}{llll|llll}
\hline Atom & Atom & Atom & Angle $\left(^{\circ}\right)$ & Atom & Atom & Atom & Angle $\left(^{\circ}\right)$ \\
\hline $\operatorname{Ti}(1)$ & $\mathrm{N}(3)$ & $\mathrm{N}(3)^{\prime}$ & $177.7(3)$ & $\mathrm{P}(1)$ & $\operatorname{Ti}(1)$ & $\mathrm{N}(3)$ & $105.24(11)$ \\
$\mathrm{P}(2)$ & $\mathrm{Ti}(1)$ & $\mathrm{P}(1)$ & $154.58(6)$ & $\mathrm{P}(1)$ & $\operatorname{Ti}(1)$ & $\mathrm{N}(2)$ & $87.19(11)$ \\
$\mathrm{N}(1)$ & $\mathrm{Ti}(1)$ & $\mathrm{N}(2)$ & $119.46(15)$ & $\mathrm{P}(1)$ & $\operatorname{Ti}(1)$ & $\mathrm{N}(1)$ & $79.30(11)$ \\
$\mathrm{P}(2)$ & $\mathrm{Ti}(1)$ & $\mathrm{N}(3)$ & $100.17(11)$ & & & & \\
$\mathrm{P}(2)$ & $\operatorname{Ti}(1)$ & $\mathrm{N}(2)$ & $80.75(11)$ & $\mathrm{N}(1)$ & $\operatorname{Ti}(1)$ & $\mathrm{N}(3)$ & $123.60(15)$ \\
$\mathrm{P}(2)$ & $\mathrm{Ti}(1)$ & $\mathrm{N}(1)$ & $87.25(11)$ & $\mathrm{N}(2)$ & $\mathrm{Ti}(1)$ & $\mathrm{N}(3)$ & $116.91(17)$ \\
\hline
\end{tabular}




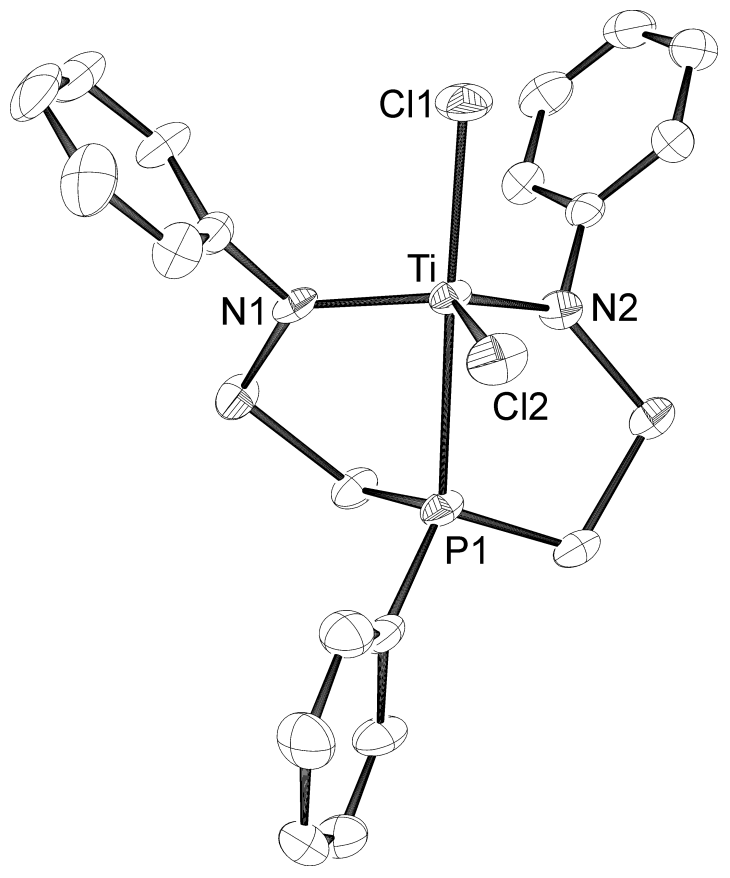

Figure S3. ORTEP representation (ellipsoid probability 50\%) of the molecular structure of $[\mathrm{NPN}] \mathrm{TiCl}_{2}$ as determined by X-ray diffraction. Hydrogen atoms and silylmethyl groups are omitted for clarity.

Table S4. Selected Bond Lengths and Angles in [NPN]TiCl 2

\begin{tabular}{lll||lll}
\hline Atom & Atom & Distance $(\AA)$ & Atom & Atom & Distance $(\AA)$ \\
\hline $\operatorname{Ti}(1)$ & $\mathrm{Cl}(1)$ & $2.2937(12)$ & $\operatorname{Ti}(1)$ & $\mathrm{N}(1)$ & $1.914(3)$ \\
$\operatorname{Ti}(1)$ & $\mathrm{Cl}(2)$ & $2.2874(12)$ & $\operatorname{Ti}(1)$ & $\mathrm{N}(2)$ & $1.936(4)$ \\
$\operatorname{Ti}(1)$ & $\mathrm{P}(1)$ & $2.6084(12)$ & & & \\
\hline
\end{tabular}

\begin{tabular}{llll||llll}
\hline Atom & Atom & Atom & Angle $\left(^{\circ}\right)$ & Atom & Atom & Atom & Angle $\left(^{\circ}\right)$ \\
\hline $\mathrm{Cl}(1)$ & $\mathrm{Ti}(1)$ & $\mathrm{P}(1)$ & $176.08(5)$ & $\mathrm{N}(2)$ & $\mathrm{Ti}(1)$ & $\mathrm{Cl}(2)$ & $119.42(11)$ \\
$\mathrm{N}(1)$ & $\mathrm{Ti}(1)$ & $\mathrm{N}(2)$ & $116.45(14)$ & $\mathrm{N}(2)$ & $\mathrm{Ti}(1)$ & $\mathrm{Cl}(1)$ & $100.45(12)$ \\
$\mathrm{N}(1)$ & $\mathrm{Ti}(1)$ & $\mathrm{Cl}(2)$ & $117.58(11)$ & $\mathrm{N}(2)$ & $\mathrm{Ti}(1)$ & $\mathrm{P}(1)$ & $75.85(11)$ \\
$\mathrm{N}(1)$ & $\mathrm{Ti}(1)$ & $\mathrm{P}(1)$ & $80.64(10)$ & $\mathrm{Cl}(2)$ & $\mathrm{Ti}(1)$ & $\mathrm{P}(1)$ & $87.80(4)$ \\
$\mathrm{N}(1)$ & $\mathrm{Ti}(1)$ & $\mathrm{Cl}(1)$ & $100.11(11)$ & $\mathrm{Cl}(2)$ & $\mathrm{Ti}(1)$ & $\mathrm{Cl}(1)$ & $95.21(5)$ \\
\hline
\end{tabular}




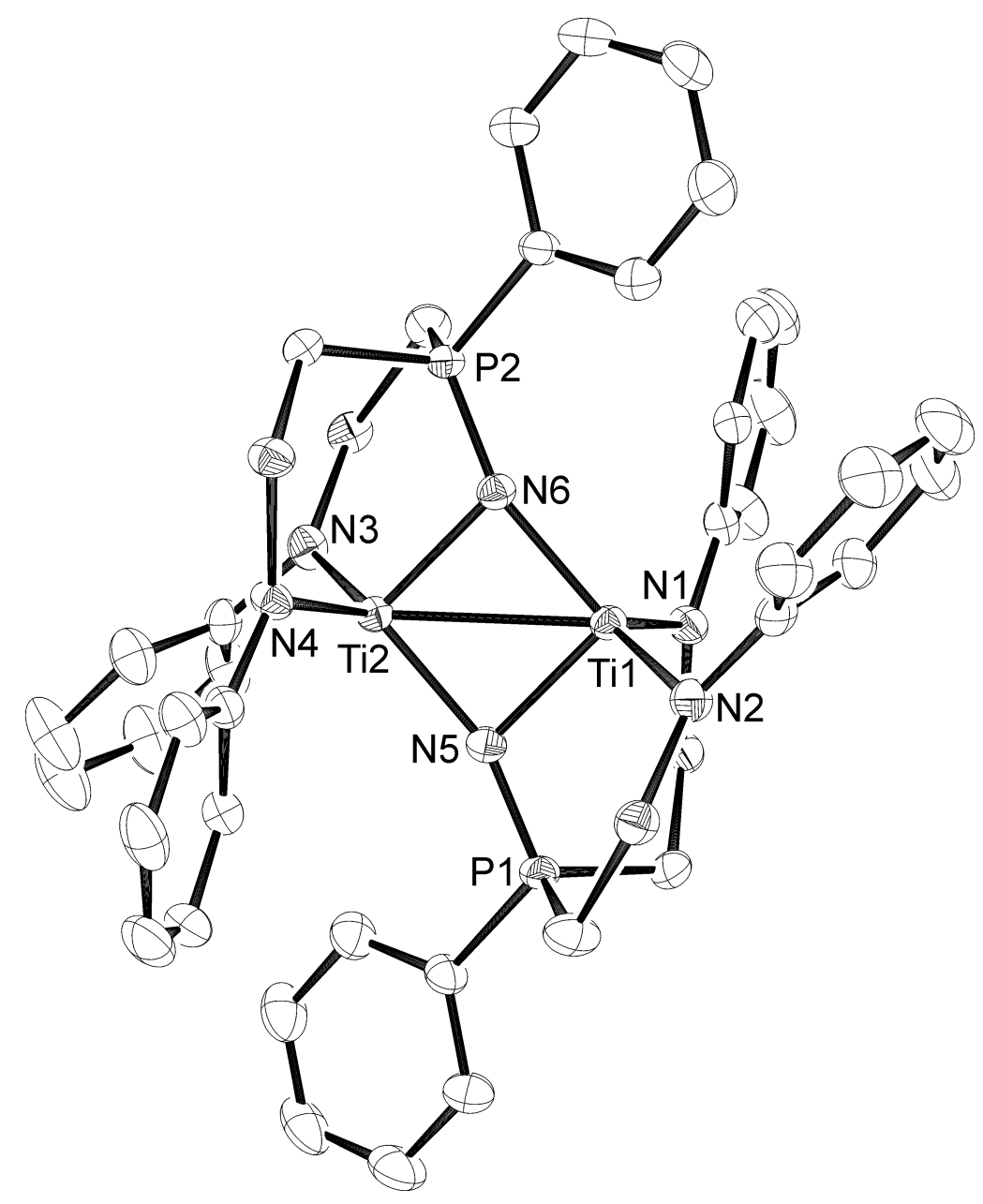

Figure S4. ORTEP representation (ellipsoid probability 50\%) of the molecular structure of $\left(\left[\mathrm{N}\left(\mathrm{N}^{\prime}\right) \mathrm{N}\right] \mathrm{Ti}\right)_{2}$ as determined by X-ray diffraction. Hydrogen atoms and silylmethyl groups are omitted for clarity.

Table S5. Selected Bond Lengths and Angles in ([N(N')N]Ti $)_{2}$

\begin{tabular}{lll||lll}
\hline Atom & Atom & Distance $(\AA)$ & Atom & Atom & Distance $(\AA)$ \\
\hline $\operatorname{Ti}(2)$ & $\operatorname{Ti}(1)$ & $2.6710(6)$ & $\mathrm{P}(1)$ & $\mathrm{N}(5)$ & $1.591(2)$ \\
$\operatorname{Ti}(2)$ & $\mathrm{N}(6)$ & $1.993(2)$ & $\operatorname{Ti}(1)$ & $\mathrm{N}(6)$ & $1.980(2)$ \\
$\operatorname{Ti}(2)$ & $\mathrm{N}(5)$ & $1.978(2)$ & $\operatorname{Ti}(1)$ & $\mathrm{N}(5)$ & $1.997(2)$ \\
$\operatorname{Ti}(2)$ & $\mathrm{N}(4)$ & $2.046(2)$ & $\operatorname{Ti}(1)$ & $\mathrm{N}(1)$ & $2.042(2)$ \\
$\operatorname{Ti}(2)$ & $\mathrm{N}(3)$ & $1.992(2)$ & $\operatorname{Ti}(1)$ & $\mathrm{N}(2)$ & $2.008(2)$ \\
$\mathrm{P}(2)$ & $\mathrm{N}(6)$ & $1.590(2)$ & & & \\
\hline
\end{tabular}

\begin{tabular}{llll||llll}
\hline Atom & Atom & Atom & Angle $\left(^{\circ}\right)$ & Atom & Atom & Atom & Angle $\left(^{\circ}\right)$ \\
\hline $\operatorname{Ti}(2)$ & $\mathrm{N}(6)$ & $\operatorname{Ti}(1)$ & $84.50(8)$ & $\mathrm{P}(2)$ & $\mathrm{N}(6)$ & $\operatorname{Ti}(1)$ & $158.18(13)$ \\
$\operatorname{Ti}(2)$ & $\mathrm{N}(5)$ & $\operatorname{Ti}(1)$ & $84.43(8)$ & $\mathrm{P}(2)$ & $\mathrm{N}(6)$ & $\operatorname{Ti}(2)$ & $112.83(11)$ \\
$\mathrm{N}(6)$ & $\operatorname{Ti}(2)$ & $\mathrm{N}(5)$ & $95.61(8)$ & $\mathrm{P}(1)$ & $\mathrm{N}(5)$ & $\mathrm{Ti}(1)$ & $112.30(12)$ \\
$\mathrm{N}(6)$ & $\mathrm{Ti}(1)$ & $\mathrm{N}(5)$ & $95.43(9)$ & $\mathrm{P}(1)$ & $\mathrm{N}(5)$ & $\mathrm{Ti}(2)$ & $161.56(14)$ \\
\hline
\end{tabular}




\section{References}

1)Pangborn, A. B.; Giardello, M. A.; Grubbs, R. H.; Rosen, R. K.; Timmers, F. J. Organometallics 1996, 15, 1518-1520.

2)Connelly, N. G.; Geiger, W. E. Chem. Rev. 1996, 96, 877-910.

3)Lalancette, J. M.; Rollin, G.; Dumas, P. Can. J. Chem. 1972, 50, 3058-3062.

4)Bergbreiter, D. E.; Killough, J. M. J. Am. Chem. Soc. 1978, 100, 2126-2134.

5)Manzer, L. E. Inorg. Synth. 1982, 21, 135-140.

6)Fryzuk, M. D.; Love, J. B.; Rettig, S. J. Chem. Commun. 1996, 2783-2784.

7)Fryzuk, M. D.; Johnson, S. A.; Patrick, B. O.; Albinati, A.; Mason, S. A.; Koetzle, T. F. J. Am. Chem. Soc. 2001, 123, 3960-3973.

8) $d^{*} T R E K$; Area Detector Software. Version 4.13. Molecular Structure Corporation, 19961998.

9)Altomare, A.; Cascarano, M.; Giacovazzo, C.; Guagliara, A. J. Appl. Cryst. 1994, 26, 343.

10)Altomare, A.; Burla, M. C.; Cammalli, G.; Cascarano, M.; Giacovazzo, C.; Guagliardi, A.; Moliterni, A. G. G.; Polidori, G.; Spagna, A. J. Appl. Cryst. 1999, 32, 115-119.

11)Beurskens, P. T.; Admiraal, G.; Beurskens, G.; Bosman, W. P.; Gelder, R. d.; Isreal, R.; Smits, J. M. M. DIRDIF94; The DIRDIF-94 program system, Technical Report of the Crystallography Laboratory: University of Nijmegen, The Netherlands, 1994. 